\title{
An optimal algorithm for preemptive on-line scheduling
}

Article in Operations Research Letters · October 1995

DOI: 10.1016/0167-6377(95)00039-9

CITATIONS

55

3 authors, including:

\section{Bo Chen}

The University of Warwick

76 PUBLICATIONS 1,413 CITATIONS

SEE PROFILE
READS

51

Some of the authors of this publication are also working on these related projects: 


\title{
An Optimal Algorithm for Preemptive On-line Scheduling *
}

\author{
Bo Chen ${ }^{\dagger}$ \\ André van Vliet $\ddagger$ \\ Gerhard J. Woeginger $\S$
}

June 22, 1995

\begin{abstract}
We investigate the problem of on-line scheduling jobs on $m$ identical parallel machines where preemption is allowed. The goal is to minimize the makespan. We derive an approximation algorithm with worst-case guarantee $m^{m} /\left(m^{m}-(m-1)^{m}\right)$ for every $m \geq 2$, which increasingly tends to $e /(e-1) \approx 1.58$ as $m \rightarrow \infty$. Moreover, we prove that for no $m \geq 2$ there does exist any approximation algorithm with a better worst-case guarantee.
\end{abstract}

Keywords. scheduling $*$ preemption $*$ on-line algorithms $*$ worst-case bounds

\section{Introduction}

We study the problem of preemptively scheduling a list $\left(J_{1}, J_{2}, \ldots\right)$ of jobs on-line on $m$ identical parallel machines. Associated with each job $J_{j}$ is its processing time (or length) $p_{j}=p\left(J_{j}\right)$. At any time, each machine can handle at most one job and each job can be processed by at most one machine. Preemption is permitted, which allows to split a job and spread its processing over several machines. The jobs are not known a priori: job $J_{j}$ becomes known only when $J_{j-1}$ has already been scheduled. As soon as job $J_{j}$ appears, it must irrevocably be assigned to one or more time slots of one or more machines. The objective is to find a schedule that minimizes the maximum completion time, or makespan.

The off-line version of this problem, where all the job information, such as the total number of jobs, their arrival times and processing times, is fully known in advance, can be easily solved to optimality in polynomial time (see McNaughton [2]). On the other hand, the off-line version without preemption is known to be strongly NP-complete [1].

The quality of an (approximation) algorithm $H$, often called heuristic, is usually measured by its worst-case (performance) ratio

$$
R^{H}(m)=\sup \{H(L) / \mathrm{Opt}(L): L \text { is a list of jobs }\},
$$

${ }^{*}$ This article appears in Operations Research Letters 18 (1995), 127-131. The work was supported by the Tinbergen Institute at Erasmus University Rotterdam and by the Christian Doppler Laboratorium für Diskrete Optimierung.

${ }^{\dagger}$ Warwick Business School, University of Warwick, Coventry, CV4 7AL, U.K.

${ }^{\ddagger}$ Econometric Institute, Erasmus University Rotterdam, 3000 DR Rotterdam, The Netherlands.

$\S$ TU Graz, Institut für Mathematik, Kopernikusgasse 24, A-8010 Graz, Austria. 
where $H(L)$ denotes the makespan of a schedule produced by heuristic $H$ for scheduling the list $L$ of jobs on $m$ machines, and $\operatorname{OpT}(L)$ denotes the corresponding makespan of some (off-line) optimal schedule.

In this note we will develop an approximation algorithm with worst-case ratio $m^{m} /\left(m^{m}\right.$ $\left.(m-1)^{m}\right)$ for on-line preemptive scheduling on $m$ machines. As $m$ increases, this ratio increasingly tends to $e /(e-1) \approx 1.58$, where $e$ denotes the Eulerian constant. By deriving a lower bound on the worst-case ratio of any on-line algorithm, we will prove that our algorithm is best possible.

\section{Preliminaries}

For a set of jobs with processing times $p_{1}, \ldots, p_{n}$, there are two straightforward lower bounds for the makespan of any preemptive schedule on $m$ machines. First, the makespan must be at least $\max _{1 \leq j \leq n}\left\{p_{j}\right\}$ since no job can be processed on two machines at the same time. Secondly, the makespan must be at least $\left(\sum_{j=1}^{n} p_{j}\right) / m$, the average machine load. McNaughton [2] proved that there always exists a schedule with makespan equal to the maximum of these two bounds.

Proposition 2.1 (McNaughton) For every set of $n$ jobs with processing times $p_{1}, \ldots, p_{n}$ and for $m \geq 1$ machines, the length OPT of the optimal preemptive schedule equals $\max \left\{\left(\sum_{j=1}^{n} p_{j}\right) / m, \max _{1 \leq j \leq n} p_{j}\right\}$.

We will use the following notation. For $m \geq 2$, we introduce the numbers $\alpha(m)=$ $m /(m-1)$ and

$$
r(m)=\frac{\alpha^{m}}{\alpha^{m}-1}=\frac{m^{m}}{m^{m}-(m-1)^{m}} .
$$

To simplify the presentation, we will drop the dependence on $m$ and always write $\alpha$ and $r$ instead of $\alpha(m)$ and $r(m)$.

\section{The Approximation Algorithm}

Throughout this section, the machine loads at step $t \geq 0$ (i.e., immediately after the $t$-th job has been scheduled) will be denoted by $\left\{L_{i}^{t}\right\}$, where $L_{1}^{t} \leq L_{2}^{t} \leq \cdots \leq L_{m}^{t}$. The corresponding machines are denoted by $M_{1}^{t}, \ldots, M_{m}^{t}$. The current optimum makespan in step $t$ (for the job set $\left.\left\{J_{1}, \ldots, J_{t}\right\}\right)$ is denoted by $\mathrm{OPT}^{t}$, and the sum $\sum_{j=1}^{t} p_{j}$ of the processing times of all known jobs is denoted by $S^{t}$.

The basic principle of our algorithm is to keep the most lightly loaded machines still lightly loaded in an anticipation of possible arrival of longer jobs. The algorithm will schedule jobs in such a way that, at any step $t \geq 0$,

(I1) $L_{m}^{t} \leq r \mathrm{OPT}^{t}$.

(I2) For any $1 \leq k \leq m$,

$$
\sum_{i=1}^{k} L_{i}^{t} \leq \frac{\alpha^{k}-1}{\alpha^{m}-1} S^{t}
$$


Let us formally describe how the algorithm schedules a new job $J_{t+1}$. First, compute the new optimum makespan $\mathrm{OPT}^{t+1}$ according to Proposition 2.1. If $L_{m}^{t}+p_{t+1} \leq r \mathrm{OPT}^{t+1}$, then schedule job $J_{t+1}$ onto machine $M_{m}^{t}$. Otherwise, let $\ell=\min \left\{1 \leq i \leq m: L_{i}^{t}+p_{t+1} \geq\right.$ $\left.r \mathrm{OpT}^{t+1}\right\}$. Schedule $r \mathrm{OpT}^{t+1}-L_{\ell}^{t}$ portion of $J_{t+1}$ onto machine $M_{\ell}^{t}$, and the remaining part of $J_{t+1}$, if any, onto machine $M_{\ell-1}^{t}$.

The following two lemmas show that the above described algorithm is well defined, and (I1) and (I2) are always fulfilled.

Lemma 3.1 If (I1) and (I2) are fulfilled at step $t$, then job $J_{t+1}$ can be successfully scheduled, and (I1) is still fulfilled at step $t+1$.

Proof. If $L_{m}^{t}+p_{t+1} \leq r \mathrm{OPT}^{t+1}$ then we are done, since $L_{m}^{t+1}=L_{m}^{t}+p_{t+1}$. Suppose $L_{m}^{t}+p_{t+1}>$ $r \mathrm{OpT}^{t+1}$. Then $\ell$ is well defined. Suppose $\ell \geq 2$. Since

$$
L_{\ell-1}^{t}+\left(p_{t+1}-\left(r \mathrm{OPT}^{t+1}-L_{\ell}^{t}\right)\right)<L_{\ell}^{t}
$$

according to the definition of $\ell$, we conclude that, if machine $M_{\ell-1}^{t}$ receives any workload of $J_{t+1}$, then it can finish the work by $L_{\ell}^{t}$, the time machine $M_{\ell}^{t}$ starts processing $J_{t+1}$. Therefore, the constraint that any job can be processed by at most one machine at a time is honored. On the other hand, we have

$$
L_{m}^{t+1}=L_{\ell}^{t}+\left(r \mathrm{OPT}^{t+1}-L_{\ell}^{t}\right)=r \mathrm{OPT}^{t+1},
$$

hence (I1) is fulfilled.

Finally, suppose $\ell=1$. We will show that $L_{1}^{t}+p_{t+1} \leq r \mathrm{OPT}^{t+1}$. Hence we can conclude that $L_{1}^{t}+p_{t+1}=r \mathrm{OpT}^{t+1}$, which simply implies what we want to prove.

Condition (I2) with $k=1$ yields $L_{1}^{t} \leq(\alpha-1) S^{t} /\left(\alpha^{m}-1\right)$. Let $S^{t}=\lambda p_{t+1}$ for some $\lambda \geq 0$. It is easy to verify that

$$
\frac{(\alpha-1) \lambda}{\alpha^{m}-1}+1 \leq r \max \left\{\frac{\lambda+1}{m}, 1\right\}
$$

which, together with the fact that

$$
\mathrm{OPT}^{t+1} \geq \max \left\{\frac{S^{t}+p_{t+1}}{m}, p_{t+1}\right\}=p_{t+1} \max \left\{\frac{\lambda+1}{m}, 1\right\},
$$

leads to

$$
\begin{aligned}
L_{1}^{t}+p_{t+1} & \leq \frac{\alpha-1}{\alpha^{m}-1} S^{t}+p_{t+1}=\left(\frac{(\alpha-1) \lambda}{\alpha^{m}-1}+1\right) p_{t+1} \\
& \leq\left(\frac{(\alpha-1) \lambda}{\alpha^{m}-1}+1\right)\left(\max \left\{\frac{\lambda+1}{m}, 1\right\}\right)^{-1} \mathrm{OPT}^{t+1} \leq r \mathrm{OPT}^{t+1}
\end{aligned}
$$

This completes our argument.

Lemma 3.2 If (I1) and (I2) are fulfilled at step $t$, then (I2) is still fulfilled at step $t+1$.

Proof. We proof that for each $1 \leq k \leq m$ the corresponding inequality in (I2) holds. Let

$$
u=\min \left\{1 \leq i \leq m: \text { machine } M_{i}^{t} \text { receives workload of } J_{t+1}\right\} .
$$


We will distinguish two cases depending on the machine index $u$.

If $k<u$, all the loads in the left-hand side of the inequality in (I2) did not increase when going from step $t$ to step $t+1$, whereas the sum of processing times in the right-hand side of (I2) did increase by $p_{t+1}$. This settles the easy first case.

In the other case $k \geq u$, we will prove that

$$
\sum_{i=k+1}^{m} L_{i}^{t+1} \geq \frac{\alpha^{m}-\alpha^{k}}{\alpha^{m}-1} S^{t+1} .
$$

Since $\sum_{i=1}^{m} L_{i}^{t+1}=S^{t+1}$, inequality (2) is equivalent to the desired inequality in (I2). If $k=m$ then (2) is trivial. Suppose $k<m$. Observe that by the way the algorithm schedules job $J_{t+1}$, we have $L_{m}^{t+1}=r \mathrm{OPT}^{t+1}$ and $L_{i}^{t+1}=L_{i+1}^{t}$ for $u<k+1 \leq i \leq m-1$, which transform the expression in the left-hand side of (2) into

$$
\sum_{i=k+2}^{m} L_{i}^{t}+r \mathrm{OPT}^{t+1} \geq \frac{\alpha^{m}-\alpha^{k+1}}{\alpha^{m}-1}\left(S^{t+1}-p_{t+1}\right)+r \mathrm{OPT}^{t+1}
$$

where the inequality follows from (I2) at step $t$ for $k+1$ and from $S^{t}=S^{t+1}-p_{t+1}$. Let $p_{t+1}=\mu S^{t+1}$ for some $0 \leq \mu \leq 1$. Then, noticing that $\operatorname{OpT}^{t+1} \geq \max \left\{S^{t+1} / m, p_{t+1}\right\}$, we conclude that the right-hand side of (3) is at least

$$
\frac{\left(\alpha^{m}-\alpha^{k+1}\right)(1-\mu)}{\alpha^{m}-1} S^{t+1}+r \max \left\{\frac{1}{m}, \mu\right\} S^{t+1} .
$$

By direct calculation one finds that the right-hand side of (4) cannot be smaller than that of $(2)$.

In conclusion, we have established the following.

Theorem 3.3 The approximation algorithm we described has a worst-case ratio at most $r(m)=m^{m} /\left(m^{m}-(m-1)^{m}\right)<e /(e-1)$.

Proof. The above two lemmas, together with the trivial fact that, at step 0, both (I1) and (I2) are fulfilled, imply that (I1) and (I2) are fulfilled at any step of the algorithm. Then (I1) immediately leads to the theorem.

\section{A Matching Lower Bound}

In this section, we prove that there cannot exist any on-line algorithm with worst-case ratio smaller than $r(m)$. From the problem statement we see that one has the freedom to reserve idle time for coming new jobs. However, intuition suggests that this sort of idle time is not necessary. The following observation supports this intuition.

Observation 4.1 If there exists an approximation algorithm $H_{1}$ for on-line preemptive scheduling with worst-case ratio $r^{*}$, then there exists another approximation algorithm $\mathrm{H}_{2}$ with a worst-case ratio at most $r^{*}$ that never introduces machine idle time between two jobs on the same machine. 
Proof. Given any schedule $S_{1}$ (with or without idle time). For any $0 \leq i \leq m$, we denote by $I_{i}$ a maximal (with respect to containment) time interval during which exactly $i$ machines are busy. Let $T_{i}=\left\{I_{i}\right\}$ and $t_{i}=\sum\left\{\left|I_{i}\right|: I_{i} \in T_{i}\right\}$ for $i=0, \ldots, m$, where $\left|I_{i}\right|$ denotes the length of interval $I_{i}$. Then we see that the makespan of $S_{1}$ is at least $\sum_{i=1}^{m} t_{i}$. Consider the following schedule $S_{2}$ : During time interval [ $\sum_{j=m-i+2}^{m} t_{j}, \sum_{j=m-i+1}^{m} t_{j}$ ] only machine $M_{i}$ up to machine $M_{m}$ are busy, $i=1, \ldots, m$. Clearly, this simple schedule does not have machine idle time between any two jobs, and its makespan is $\sum_{i=1}^{m} t_{i}$. Moreover, both schedules $S_{1}$ and $S_{2}$ have the same $\left\{t_{i}\right\}_{i=0}^{m}$.

Now suppose both $S_{1}$ and $S_{2}$ are schedules of the same set of jobs, and one more job is added to $S_{1}$. Suppose the job is processed within time interval(s) of $T_{i}$ for $q_{i}$ time units, $i=0, \ldots, m-1$. Then in $S_{2}$ we can simulate this assignment by letting machine $M_{m-i}$ start processing $q_{i}$ portion of the job at time $\sum_{j=i+1}^{m} t_{j}, i=0, \ldots, m-1$. It is easy to see that after adding the job to $S_{1}$ and $S_{2}$ in the above way, the makespan of the new $S_{2}$ is still less than or equal to that of the new $S_{1}$, and both new schedules have the same new $\left\{t_{i}\right\}_{i=1}^{m}$.

It should be clear now that the required approximation algorithm $H_{2}$ can be obtained by making $H_{2}$ simulate $H_{1}$ for assigning every job in the way we just described. Both $H_{1}$ and $\mathrm{H}_{2}$ start with the same empty schedule.

Next, we introduce the following sequence of jobs, and consider any algorithm's behavior in assigning these jobs.

- At step 0 , there arrive $m$ jobs, all with processing times $1 / m$.

- At step $t, t \geq 1$, there arrives a new job $J_{t}$ with processing time $p_{t}=m^{t-1} /(m-1)^{t}$.

Observation 4.2 At step $t$, immediately after the arrival of job $J_{t}$, the overall sum of processing times equals $\alpha^{t}$ and the optimum makespan equals $p_{t}=\alpha^{t} / \mathrm{m}$.

Proof. This follows from Proposition 2.1.

Now suppose that there exists an approximation algorithm $H$ with worst case ratio $r(m)-\varepsilon$ for some real $\varepsilon>0$. By Observation 4.1 we may assume that $H$ never introduces machine idle time. Analogously to the preceding section, we denote by $L_{1}^{t} \leq L_{2}^{t} \leq \cdots \leq L_{m}^{t}$ the machine loads at the time immediately after algorithm $H$ scheduled job $J_{t}$.

Claim 4.3 For any $t \geq 0, L_{m}^{t} \leq(r-\varepsilon) \alpha^{t} / m$ must hold.

Proof. Since $L_{m}^{t} \leq(r-\varepsilon) \mathrm{OPT}^{t}=(r-\varepsilon) \alpha^{t} / m$.

Claim 4.4 For all $1 \leq i \leq m-1$ and all $t \geq 0, L_{m-i}^{t+i} \leq L_{m}^{t}$ holds.

Proof. We use induction on $i$. For $i=1$ we must prove that $L_{m-1}^{t+1} \leq L_{m}^{t}$ holds. Suppose the contrary. Then at step $t+1$, two machines have loads larger than $L_{m}^{t}$. Since $H$ does not introduce machine idle time, at the time point $L_{m}^{t}$, the job $J_{t+1}$ was processed on at least two machines with largest and second largest load, which is a contradiction.

Now assume that we have proved the claim up to some fixed value $i(1 \leq i \leq m-2)$, i.e., $L_{m-i}^{t+i} \leq L_{m}^{t}$. In case $L_{m-i-1}^{t+i+1}>L_{m}^{t} \geq L_{m-i}^{t+i}$ would be true, we could argue as above that job $J_{t+i+1}$ is processed on at least two machines at the same time. 
Theorem 4.5 For all $m \geq 2$, there does not exist any on-line algorithm for preemptive scheduling on $m$ machines with worst-case ratio strictly smaller than $r(m)$.

Proof. We are ready to derive a contradiction to the assumption that $H$ has worst-case ratio $r-\varepsilon$. Let us take a closer look at step $(m-1)$.

By Claim 4.4, we know that $L_{i}^{m-1} \leq L_{m}^{i-1}$ for all $1 \leq i \leq m-1$, which, together with Claim 4.3 and Observation 4.2, yields that $(r-\varepsilon)$ times the current optimum makespan

$$
\begin{aligned}
(r-\varepsilon) \alpha^{m-1} / m & \geq L_{m}^{m-1}=\alpha^{m-1}-\sum_{i=1}^{m-1} L_{i}^{m-1} \\
& \geq \alpha^{m-1}-\sum_{j=1}^{m-1} L_{m}^{j-1} \geq \alpha^{m-1}-\sum_{i=1}^{m-1}(r-\varepsilon) \alpha^{i-1} / m
\end{aligned}
$$

Comparing the last expression in the right-hand side to the left-hand side and doing some easy calculations, we see that $\varepsilon \leq 0$ must hold.

Acknowledgement. The main part of this work was carried out while the first author was working at Erasmus University Rotterdam, and the third author was visiting this University.

\section{References}

[1] M.R. Garey and D.S. Johnson, "Strong NP-completeness results: Motivation, examples and implications", J. Assoc. Comput. Mach. 25 (1978), 499-508.

[2] R. McNaughton, "Scheduling with deadlines and loss functions", Management Sci. 6 (1959), 1-12. 